\title{
Naringenin alleviates myocardial ischemia reperfusion injury by enhancing the myocardial miR-126-PI3K/AKT axis in streptozotocin-induced diabetic rats
}

\author{
SHANG-HAI LI ${ }^{1 *}$, MING-SHUANG WANG ${ }^{2 *}$, WEI-LIANG KE ${ }^{1}$ and MING-RUI WANG ${ }^{3}$ \\ ${ }^{1}$ Department of Cardiology, The Affiliated Hospital of Guangdong Medical University, Zhanjiang, Guangdong 524000; \\ ${ }^{2}$ First Operating Room, The First Affiliated Hospital of Jilin University, Changchun, Jilin 130000; \\ ${ }^{3}$ Department of Obstetrics and Gynecology, The Affiliated Hospital of Guangdong Medical University, \\ Zhanjiang, Guangdong 524000, P.R. China
}

Received August 14 2019; Accepted September 15, 2020

DOI: $10.3892 /$ etm.2021.10242

\begin{abstract}
Ischemic heart disease (IHD) is a leading cause of death in patients with type 1 diabetes. The key to treating IHD is to restore blood supply to the ischemic myocardium, which inevitably causes myocardial ischemia reperfusion (MI/R) injury. Although naringenin (Nar) prevents MI/R injury, the role of Nar in diabetic MI/R (D-MI/R) injury remains to be elucidated. The PI3K/AKT signaling pathway and microRNA (miR)-126 have previously been shown to serve anti-MI/R injury roles. The present study aimed to investigate the protection of Nar against D-MI/R injury and the role of the miR-126-PI3K/AKT axis. Diabetic rats were treated distilled water or Nar (25 or $50 \mathrm{mg} / \mathrm{kg}$, orally) for 30 days and then exposed to MI/R. The present results revealed that Nar alleviated MI/R injury in streptozotocin (STZ)-induced diabetic rats, as shown below: the reduction myocardial enzymes levels was measured using spectrophotometry, the increase of cardiac viability was detected by MTT assay, the inhibition of myocardial oxidative stress was measured using spectrophotometry and the enhancement of cardiac function were recorded using a hemodynamic monitoring system. Furthermore, Nar upregulated the myocardial miR-126-PI3K/AKT axis in D-MI/R rats. These results indicated that Nar alleviated MI/R injury through upregulating the myocardial miR-126-PI3K/AKT axis in STZ-induced diabetic rats. The current findings revealed that Nar, as an effective agent against D-MI/R injury, may provide an effective approach in the management of diabetic IHD.
\end{abstract}

Correspondence to: Professor Wei-Liang Ke, Department of Cardiology, The Affiliated Hospital of Guangdong Medical University, 57 People's Road South, Zhanjiang, Guangdong 524000, P.R. China E-mail: 182323@qq.com

*Contributed equally

Key words: ischemic heart disease, myocardial ischemia reperfusion injury, naringenin, PI3K/AKT signaling, microRNA-126

\section{Introduction}

Ischemic heart disease (IHD) is a threat to human health $(1,2)$, and the key to treating IHD is to restore blood supply to the ischemic myocardium as soon as possible (3). However, restoration of blood supply is inevitably accompanied by subsequent cardiac injury, which is widely recognized as myocardial ischemia reperfusion $(\mathrm{MI} / \mathrm{R})$ injury $(1,4,5)$. Since IHD became the leading cause of death in patients with type 1 diabetes (6), the MI/R injury in diabetes has attracted widespread attention. Epidemiological data have demonstrated that the incidence rate of ischemic cardiomyopathy among patients with diabetes is $\sim 4$-fold higher than that among patients without diabetes (7). Patients with diabetes are more susceptible to $\mathrm{MI} / \mathrm{R}$ injury $(8,9)$, with worse clinical prognosis and higher fatality rate compared with patients without diabetes $(10,11)$. Therefore, it is necessary to implement new strategies to prevent MI/R injury in patients with diabetes to improve the effectiveness diabetic IHD treatment.

Naringenin (Nar) is a naturally occurring flavanone, predominantly derived from citrus fruits. Nar plays a biological role in the human body (12), and is known to exert cardioprotective effects (13). Nar prevents doxorubicin-induced toxicity, including apoptosis and oxidative stress in cardiomyoblasts (14-17). In addition, Nar antagonizes hypercholesterolemia-induced cardiac oxidative stress and subsequent necroptosis in rats (18). Although a few studies have confirmed that Nar is able to attenuate MI/R injury $(19,20)$, to the best of our knowledge, whether Nar counteracts MI/R injury in diabetes has not yet been clarified. It has been reported that Nar protects cardiomyocytes against hyperglycemia-induced injury (21). Therefore, in the present study, streptozotocin (STZ)-induced diabetic rats were exposed to $\mathrm{MI} / \mathrm{R}$ to construct a model of diabetic MI/R (D-MI/R) injury, and to explore whether Nar has a potential therapeutic function.

The PI3K/AKT signaling pathway plays a critical protective role in MI/R injury (22). In the myocardium, the PI3K/AKT signaling pathway indirectly regulates the contraction of the cardiac muscle and the function of calcium channels (23). 
Accumulating evidence has demonstrated that the PI3K/AKT signaling pathway is impaired during MI/R injury, and that $\mathrm{MI} / \mathrm{R}$ injury can be ameliorated by activation of the PI3K/AKT signaling pathway (24-26). These previous studies provided evidence that activated PI3K/AKT signaling may improve MI/R injury. Therefore, the present study investigated whether the PI3K/AKT signaling pathway is involved in the protective effect of Nar against MI/R injury in diabetes.

MicroRNA-126 (miR-126) is involved in the pathophysiological processes of various cardiovascular diseases, particularly MI/R injury (27-29). It has been demonstrated that miR-126 attenuates oxidative stress and apoptosis induced by $\mathrm{MI} / \mathrm{R}$ injury in rats $(27,28)$. Notably, miR-126 may be a therapeutic agent for diabetes mellitus (30). Diabetes reduces miR-126 expression and angiogenesis in the myocardium compared with healthy rats, while enhancement of myocardial miR-126 level significantly improves myocardial angiogenesis in diabetic rats (31). Therefore, although an association between Nar and miR-126 has not been reported to date, the aforementioned studies led to a hypothesis that miR-126 may be involved in the protective effects of Nar against MI/R injury in diabetes.

The present study aimed to explore whether Nar antagonizes MI/R-injury in diabetic rats and whether miR-126-PI3K/AKT are involved in this protective effect. The results of the present study demonstrated that Nar significantly ameliorated MI/R injury and enhanced the myocardial miR-126-PI3K/AKT axis in rats with $\mathrm{D}-\mathrm{MI} / \mathrm{R}$. The results suggest that Nar alleviated $\mathrm{MI} / \mathrm{R}$ injury via enhancing the myocardial miR-126-PI3K/AKT axis in STZ-induced diabetic rats.

\section{Materials and methods}

Reagents. STZ, Nar, ketamine, xylazine and an MTT kit were supplied by Sigma-Aldrich; Merck KGaA. The BCA protein assay kit was obtained from Dojindo Molecular Technologies, Inc. Lactate dehydrogenase (LDH) and creatine kinase myocardial band (CK-MB) measurement kits were obtained from the Nanjing Jiancheng Bioengineering Institute. The assay kits for glutathione peroxidase (GSH-Px), superoxide dismutase (SOD), malondialdehyde (MDA), 8-hydroxy-2 deoxyguanosine (8-OHdG) and $\mathrm{H}_{2} \mathrm{O}_{2}$ measurement were purchased from Wuhan USCN Business Co., Ltd. Specific monoclonal antibodies against phosphorylated (p)-PI3K (cat. no. 4228), PI3K (cat. no. 4255), p-AKT (cat. no. 9271), AKT (cat. no. 9272) and GAPDH (cat. no. 2118), Anti-rabbit IgG-HRP-linked Antibody (cat. no. 7074) were supplied by Cell Signaling Technology, Inc.

Animals. A total of 50 adult male Sprague-Dawley rats (age, 8-10 weeks; weight, $250 \pm 10 \mathrm{~g}$ ) were supplied by Guangdong Medical Laboratory Animal Center. Rats were raised in single cages (individually) in a specific-pathogen-free environment under a 12-h light/dark cycle (light exposure, 7:00 a.m.-7:00 p.m.), and maintained at a constant temperature $\left(22 \pm 2^{\circ} \mathrm{C}\right)$, ventilation and humidity $(50 \%)$. Rats were provided with food and water ad libitum, and the bedding was changed 1-2 times a week to ensure a suitable environment for the rats.

Induction of diabetes. STZ was dissolved in $0.1 \mathrm{~mol} / 1$ sodium citrate buffer ( $\mathrm{pH} 4.3$ ) and used immediately after preparation, which was performed in a cold $\left(4-6^{\circ} \mathrm{C}\right)$ and dark environment. After overnight (12-h) fasting, the rats were weighed. The STZ-treated groups received a single intraperitoneal (i.p.) injection of STZ $(55 \mathrm{mg} / \mathrm{kg})$, while the other groups received an equivalent dose of PBS (i.p.). The rats were administered food $1 \mathrm{~h}$ after STZ injection. A total of $72 \mathrm{~h}$ after the STZ injection, Baseline body weight and blood glucose level were measured in all the rats. After STZ or PBS injection, blood glucose levels at day 3 and day 30 were determined measured using a glucometer (Roche Applied Science), and body weights were measured once a week. Only animals with fasting blood glucose levels $>16.7 \mathrm{mmol} / 1$ at 3 days after STZ injection were considered diabetic and $\sim 80$ rats used for further experiments (32). Finally, six of the rats did not reach their target levels of blood glucose, which were supplemented with STZ $(10-20 \mathrm{mg} / \mathrm{kg}) 3$ days later. Failing that, diabetic induction was repeated from scratch once blood glucose levels had returned to normal.

Experimental protocol. After a week of adaptation to the experimental environment, then 3 days after the STZ or PBS injection, the rats were randomly divided into the following five groups (10 rats in each group) and were provided with the following treatments: i) Control-sham group (C-S), non-diabetic rats that received sham surgery; ii) diabetes-sham group (D-S), diabetic rats that received sham surgery; iii) D-MI/R group, diabetic rats that were orally treated with vehicle (distilled water, $1.5 \mathrm{ml}$ ) for 30 days, and then subjected to MI/R injury; iv) D-MI/R + low-dose Nar (LN) group, diabetic rats that were orally treated with $\operatorname{Nar}(25 \mathrm{mg} / \mathrm{kg} / \mathrm{day}$; diluted in distilled water) for 30 days and then subjected to I/R injury; and v) D-MI/R + high-dose Nar (HN) group, diabetic rats that were orally treated with $\operatorname{Nar}(50 \mathrm{mg} / \mathrm{kg} / \mathrm{day}$; diluted in sterile water) for 30 days and then subjected to I/R injury.

$M I / R$ modeling. After the aforementioned 4 weeks of treatment with Nar or distilled water, the hearts of the rats were isolated and perfused according to a previously described protocol $(33,34)$. Briefly, the each rat were heparinized (500 IU) to avoid blood clotting during surgery and then anesthetized with ketamine (60 mg/kg; i.p.) and xylazine (10 mg/kg; i.p.). Subsequently, the hearts were rapidly excised and immediately perfused on a Langendorff device (ADInstruments, Ltd.) with modified Krebs-Henseleit solution $\left(\mathrm{pH} 7.4 ; 37^{\circ} \mathrm{C}\right)$ containing $118 \mathrm{mmol} / 1 \mathrm{NaCl}, 4.7 \mathrm{mmol} / 1 \mathrm{KCl}, 1.25 \mathrm{mmol} / 1 \mathrm{CaCl}_{2}$, $1.2 \mathrm{mmol} / 1 \mathrm{MgSO}_{4}, 25 \mathrm{mmol} / 1 \mathrm{NaHCO}_{3}, 1.2 \mathrm{mmol} / 1 \mathrm{KH}_{2} \mathrm{PO}_{4}$ and $11 \mathrm{mmol} / \mathrm{l}$ glucose, saturated with $95 \% \mathrm{O}_{2}$ and $5 \% \mathrm{CO}_{2}$. Upon stabilization, the hearts were subjected to 30-min regional ischemia by tightening a 5-0 silk suture around the left anterior descending coronary artery and reperfusion for $120 \mathrm{~min}$. The sham groups underwent sham surgery (the left anterior descending coronary artery was not ligated) in which the heart was exteriorized without MI/R injury.

Evaluating myocardial systolic and diastolic functions. For measurement of interventricular pressure changes, a water-filled latex balloon was inserted into the left ventricle, and the signals were delivered to the associated transducer through a connecting pressure catheter. After reperfusion, the cardiac function index, including left ventricular systolic pres- 
sure (LVSP), left ventricular end-diastolic pressure (LVEDP) and left ventricular maximal systolic/diastolic velocity $\left( \pm \mathrm{dP}^{2} \mathrm{dt}_{\max },+\mathrm{dP} / \mathrm{dt}_{\max }=\right.$ the maximum rate of pressure rise in the left ventricle; $-\mathrm{dP} / \mathrm{dt}_{\max }=$ the maximum rate of pressure drop in the left ventricle), were recorded using a hemodynamic monitoring system (PowerLab-PL3508; ADInstruments, Ltd.).

Measurement of cardiac tissue viability, and $C K$ and $L D H$ levels. After reperfusion, the hearts were cut into 2-mm-thick sections and incubated with MTT $(3 \mathrm{mM})$ at $37^{\circ} \mathrm{C}$ for $30 \mathrm{~min}$ to form formazan (34). Based on previous study, cardiac tissue viability was measured by the level of formazan using a spectrophotometer at $550 \mathrm{~nm}$ (34). In addition, at $5 \mathrm{~min}$ after reperfusion, the coronary effluent was collected for the determination of cardiac CK-MB and LDH activities, which are two major indicators of MI/R injury, by spectrophotometry using the aforementioned commercial assay kits according to a previously described protocol (35). The activities of these two enzymes were expressed as $\mathrm{U} / \mathrm{l}$.

Mitochondrial oxidative stress quantification. After reperfusion, the myocardial tissue was homogenized $(10 \% \mathrm{w} / \mathrm{v})$ in $0.1 \mathrm{~mol} / 1 \mathrm{PBS}$ and centrifuged at $12,000 \mathrm{x}$ g for $10 \mathrm{~min}$ at $4{ }^{\circ} \mathrm{C}$. Subsequently, the supernatant was collected, and the protein concentration was quantified using the aforementioned BCA protein assay. As previously described $(36,37)$, the activities of GSH-Px and SOD, the levels of MDA and 8-OHdG, and $\mathrm{H}_{2} \mathrm{O}_{2}$ formation in the supernatant were determined by spectrophotometry using the aforementioned commercially available kits.

Western blot analysis. The protein levels of p-PI3K, PI3K, p-AKT and AKT were measured by western blotting. After reperfusion, myocardial tissue was homogenized in ice-cold homogenizing buffer [20 mM Tris-Cl (pH 7.4), $150 \mathrm{mM} \mathrm{NaCl}$, $1 \mathrm{mM}$ EDTA, $1 \%$ Triton X-100 and $1 \mathrm{mM}$ phenylmethanesulfonyl fluoride). After centrifugation at 12,000 x g for $30 \mathrm{~min}$ at $4^{\circ} \mathrm{C}$, the supernatant was collected and the protein concentration was analyzed using a BCA protein assay kit (Beyotime Institute of Biotechnology). Approximately $10 \mu \mathrm{g}$ of protein for each sample were resolved by SDS-PAGE (8-12\%) and transferred onto PVDF membranes by electroblotting. Non-specific protein binding was blocked with TBS-Tween-20 [TBS-T; $50 \mathrm{mmol} / 1 \mathrm{Tris}-\mathrm{HCl}$ (pH 7.5), $150 \mathrm{mmol} / 1 \mathrm{NaCl}$ and $0.05 \%$ Tween-20] containing 5\% non-fat milk for $2 \mathrm{~h}$ at room temperature, and the membranes were incubated overnight at $4^{\circ} \mathrm{C}$ with the aforementioned primary antibodies (P-PI3K, PI3K, P-AKT, AKT, GAPDH; dilution, 1:1,000). After the membranes were washed three times with TBS-T buffer, the blots were incubated with a horseradish peroxidase-conjugated anti-rabbit secondary antibody $(1: 5,000)$ for $2 \mathrm{~h}$ at $4^{\circ} \mathrm{C}$. Subsequently, the membranes were washed with TBS-T buffer (five times for 5 min each) and electrogenerated chemiluminescence reaction solution (SuperSignal ${ }^{\mathrm{TM}}$ West Pico PLUS Chemiluminescent Substrate-A38555; Thermo Fisher Scientific, Inc.) was added for $30 \mathrm{sec}$. The protein bands were visualized using a Tanon-5600 Imaging System (Tanon Science and Technology Co., Ltd.). The semi-quantitative analysis of each blot was performed using SigmaScan Pro 5.0 software (Systat Software, Inc) and expression values were normalized to that of GAPDH.
Reverse transcription-quantitative PCR (RT-qPCR). The myocardial miR-126 level was measured by RT-qPCR as previously described (28). Briefly, total RNA from the myocardial tissue of rats was isolated using the TRIzol ${ }^{\circledR}$ reagent (Thermo Fisher Scientific, Inc.). Next, miRNA-specific stem-loop primers were reverse transcribed to cDNA according to the instructions of the miRNA RT kit (Takara Biotechnology Co., Ltd.). RT reaction conditions were processed at $42^{\circ} \mathrm{C}$ for $15 \mathrm{~min}$, followed by $3 \mathrm{~min}$ at $95^{\circ} \mathrm{C}$. qPCR was performed using SYBR Premix Ex Taq ${ }^{\mathrm{TM}}$ according to the manufacturer's instructions (Takara Biotechnology Co., Ltd.). The following PCR protocol was used: $95^{\circ} \mathrm{C}$ for $10 \mathrm{~min}$, followed by 40 cycles of $95^{\circ} \mathrm{C}$ for $15 \mathrm{sec}$ and $60^{\circ} \mathrm{C}$ for $1 \mathrm{~min}$. The $2^{-\Delta \Delta \mathrm{Cq}}$ method (38) was used to calculate the relative expression levels of miR-126 and U6 was used as an internal reference. The sequences of the primers (Beyotime Institute of Biotechnology) used were as follows: miR-126 forward, 5'-ACTGTCACTCTCATCACAAGCGC-3' and reverse, 5'-ACGCTGGCTCAGGGATCAGAGA-3'; and U6 forward, 5'-CTCGCTTCGGCAGCACA-3' and reverse, 5'-AACGCTTCACGAATTTGCGT-3'.

Statistical analysis. All experiments were repeated $\geq 3$ times, and statistical analyses were performed using SPSS 20.0 software (IBM Corp.). Data are presented as the mean \pm SEM, and differences between groups were assessed using one-way analysis of variance and Tukey's post hoc test. Repeated measurement data were analyzed using mixed ANOVA and a Tukey's post-hoc test for simple main effects. Two-tailed $\mathrm{P}<0.05$ was considered to indicate a statistically significant difference.

\section{Results}

Nar does not affect blood glucose levels or body weight in STZ-induced diabetic rats. Blood sugar levels and weight were regularly measured in diabetic rats to determine whether Nar had an effect on these parameters. The blood glucose level of the diabetic groups was significantly higher than that of the control group 3 days after STZ injection (Fig. 1A). At day 30, the blood glucose levels in the four diabetic groups remained high, and there was no significant difference in blood glucose levels between STZ-treated rats and rats co-treated with STZ and Nar (Fig. 1A).

Body weight was measured once a week after STZ or PBS injection. The body weight of STZ-induced diabetic rats was significantly reduced compared with that of the control group. In addition, there were no significant differences in body weight between STZ-treated rats and rats co-treated with STZ and Nar (Fig. 1B). These data indicate that STZ injection resulted in a diabetic status in rats, and that Nar did not affect the blood glucose level or body weight of diabetic rats.

Nar decreases myocardial enzyme content and enhances cardiac tissue viability in $D-M I / R$ rats. To determine if Nar alleviated MI/R injury in diabetic rats, the present study first explored whether Nar decreased myocardial enzyme levels and enhanced cardiac tissue viability by observing the effects of Nar on the levels of LDH and CK-MB in the coronary effluent, and the viability of heart tissues in D-MI/R rats. 

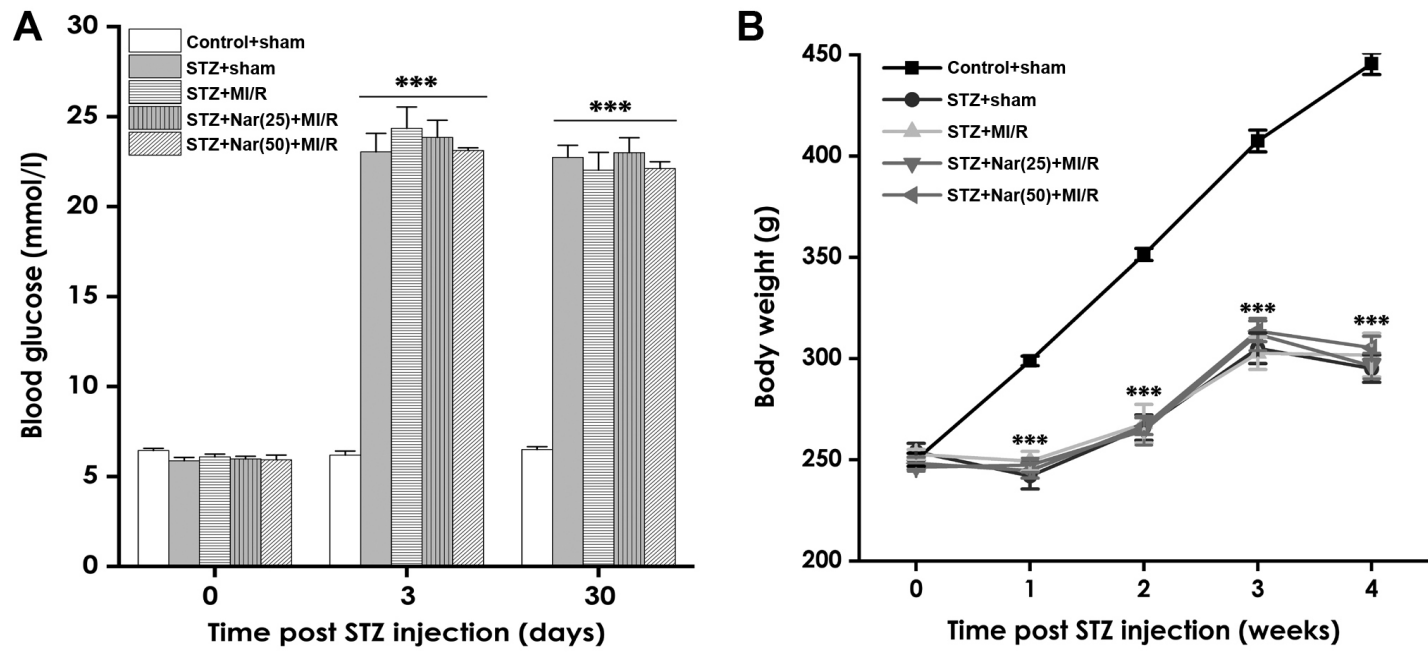

Figure 1. Effect of Nar on body weight and blood glucose levels in rats. (A) Blood glucose levels at days 0,3 and 30 were determined with a blood glucose meter. (B) Body weight in the different groups was measured once a week. Data are presented as the mean \pm SEM (number of repeated measurements: $n=8$-10/group). ${ }^{* * *} \mathrm{P}<0.001$ compared with the control-sham group. Nar, naringenin; STZ, streptozotocin; MI/R, myocardial ischemia reperfusion.


Figure 2. Effect of Nar on myocardial enzyme levels and cardiac viability in diabetic MI/R rats. Levels of (A) LDH and (B) CK-MB in the coronary effluent were detected using spectrophotometry by commercial assay kits. (C) Cardiac formazan content was measured using an MTT assay. Data are presented as the mean \pm SEM (number of repeated measurements: $\mathrm{n}=3$ /group). ${ }^{* * *} \mathrm{P}<0.01$ and ${ }^{* * * *} \mathrm{P}<0.001$ compared with the STZ-sham group; ${ }^{*} \mathrm{P}<0.05$ and ${ }^{\# \# /} \mathrm{P}<0.01$ compared with the STZ-M/IR group. LDH, lactate dehydrogenase; CK-MB, creatine kinase myocardial band; STZ, streptozotocin; MI/R, myocardial ischemia reperfusion; Nar, naringenin.

The results showed that HN significantly downregulated the levels of LDH and CK-MB (Fig. 2A and B) in the coronary effluent, and increased the cardiac formazan content (Fig. 2C) in D-MI/R rats compared with that in the untreated D-MI/R group. Moreover, there was no significant difference in the levels of LDH, CK-MB or formazan content between the $\mathrm{C}-\mathrm{S}$ and D-S groups. These data indicate that Nar decreased the myocardial enzyme content and increased cardiac tissue viability in $\mathrm{D}-\mathrm{MI} / \mathrm{R}$ rats.

Nar inhibits myocardial oxidative stress in D-MI/R rats. To confirm that Nar alleviates MI/R injury in diabetic rats, the present study investigated whether Nar antagonized myocardial oxidative stress by observing the effects of Nar on the activity of GSH-Px and SOD, and the levels of MDA, 8-OHdG and $\mathrm{H}_{2} \mathrm{O}_{2}$ in the myocardium of D-MI/R rats. In the D-MI/R group, upregulation in the content of MDA, 8- $\mathrm{OHdG}$ and $\mathrm{H}_{2} \mathrm{O}_{2}$, and downregulation in the activities of GSH-Px and SOD in the myocardium of D-MI/R rats were observed, compared with the D-S group, while these effects were markedly reversed by pretreatment with Nar (Fig. 3A-E). Furthermore, there was no significant difference in myocardial oxidative stress indexes between the C-S and D-S groups. These data indicated that Nar inhibited myocardial oxidative stress in D-MI/R rats (Fig. 3A-E).

Nar improves cardiac function in D-MI/R rats. To confirm that Nar alleviates MI/R injury in diabetic rats, the present study sought to determine whether Nar ameliorated cardiac function by observing the effects of Nar on left ventricular hemodynamic parameters in $\mathrm{D}-\mathrm{MI} / \mathrm{R}$ rats. As shown in Fig. 4, compared with the D-S group, the D-MI/R group had lower LVSP and $\pm \mathrm{dP} / \mathrm{dt}_{\max }$, as well as higher LVEDP. However, pretreatment with $\mathrm{HN}$ for 30 days significantly increased LVSP and $\pm \mathrm{dP} / \mathrm{dt}_{\max }$, and reduced LVEDP in D-MI/R rats (Fig. 4A-D). In addition, there were no significant differences in LVSP, LVEDP or $\pm \mathrm{dP} / \mathrm{dt}_{\max }$ between the C-S and $\mathrm{D}-\mathrm{S}$ groups. These data indicate that Nar alleviated cardiac dysfunction in D-MI/R rats (Fig. 4A-D).

Nar upregulates myocardial PI3K/AKT signaling in D-MI/R rats. To explore whether the PI3K/AKT signaling pathway was involved in the Nar-mediated protection against MI/R injury in diabetic rats, the present study determined whether 
A

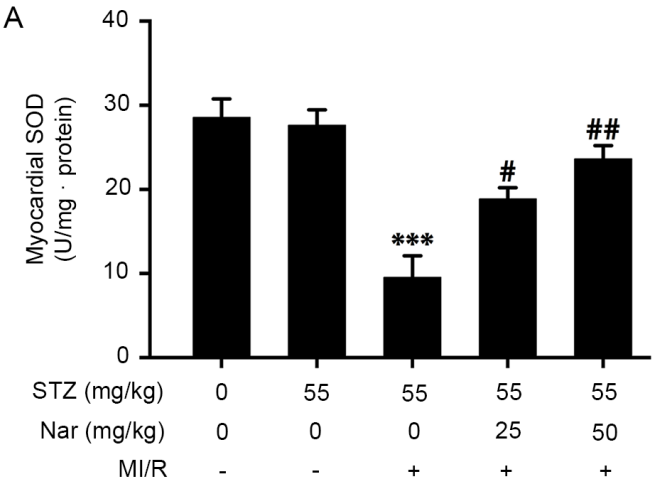

C

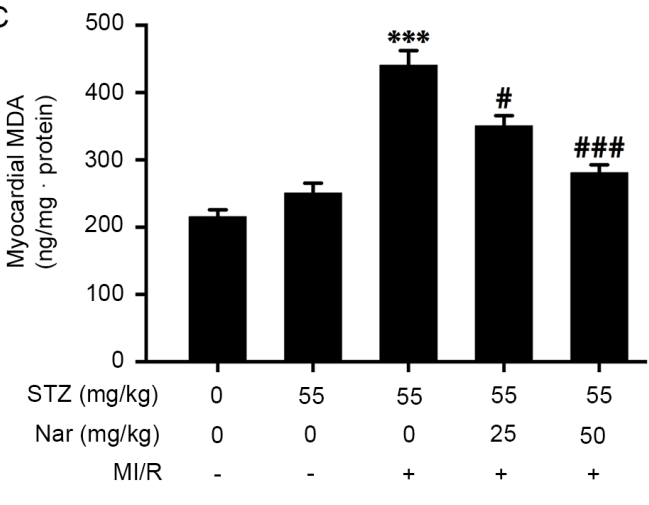

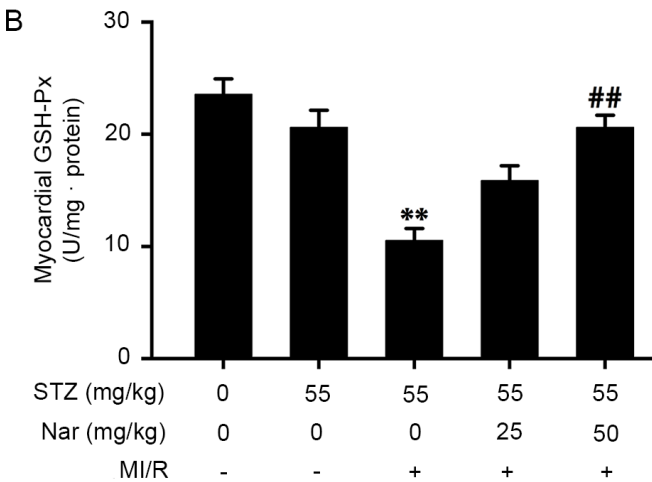

D

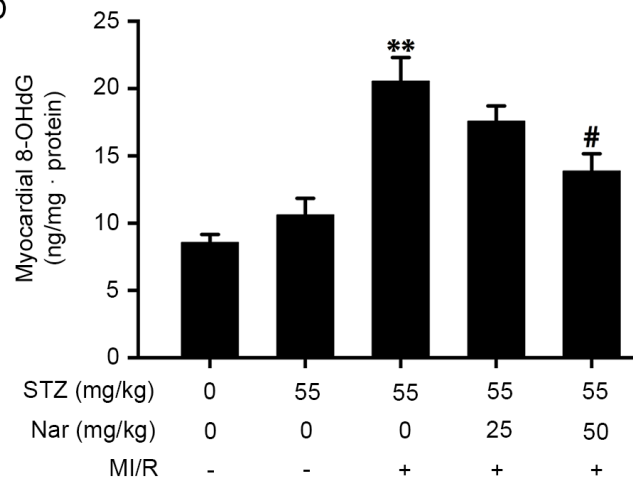

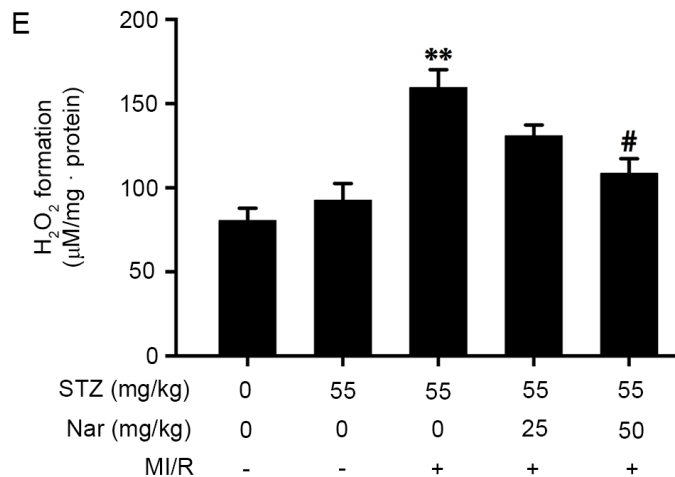

Figure 3. Effect of Nar on myocardial oxidative stress in diabetic MI/R rats. Activities of (A) SOD and (B) GSH-Px, and the levels of (C) MDA, (D) 8-OHdG as well as (E) $\mathrm{H}_{2} \mathrm{O}_{2}$ formation in the myocardium were determined by spectrophotometry using commercially available kits. Data are presented as the mean \pm SEM (number of repeated measurements: $\mathrm{n}=3$ /group). ${ }^{* *} \mathrm{P}<0.01$ and ${ }^{* * * *} \mathrm{P}<0.001$ compared with the STZ-sham group; ${ }^{*} \mathrm{P}<0.05$, ${ }^{\# \#} \mathrm{P}<0.01$ and ${ }^{\# \# \#} \mathrm{P}<0.001$ compared with the STZ-M/IR group. GSH-Px, glutathione peroxidase; SOD, superoxide dismutase; MDA, malondialdehyde; 8-OHdG, 8-hydroxy-2 deoxyguanosine; STZ, streptozotocin; MI/R, myocardial ischemia reperfusion; Nar, naringenin.

Nar regulated PI3K/AKT signaling. As represented in Fig. 5, the ratios of $\mathrm{p}-\mathrm{PI} 3 \mathrm{~K} / \mathrm{PI} 3 \mathrm{~K}$ and $\mathrm{p}-\mathrm{AKT} / \mathrm{AKT}$ were significantly downregulated in D-MI/R rats, while pretreatment with Nar markedly abolished this downregulation (Fig. 5), indicating that enhanced PI3K/AKT signaling may contribute to the Nar-mediated protection against MI/R injury in diabetic rats.

Nar upregulates myocardial miR-126 expression in D-MI/R rats. To investigate whether miR-126 was involved in the Nar-mediated protection against MI/R injury in diabetic rats, the present study examined the effect of Nar on myocardial miR-126 expression in D-MI/R rats. Myocardial miR-126 expression was significantly lower in D-MI/R rats compared with that in the D-S group, while HN significantly reversed this downregulation of miR-126 in D-MI/R rats (Fig. 6), indicating that the upregulation of miR-126 may participate in the Nar-mediated protection against MI/R injury in diabetic rats.

\section{Discussion}

The anti-MI/R injury role of Nar has been previously reported $(19,20,39)$. Activation of PI3K/AKT signaling alleviates MI/R injury (24-26), and enhanced miR-126 prevents MI/R injury $(27,28)$. The present study used STZ-induced diabetic rats to investigate whether Nar could ameliorate MI/R injury in diabetes, and to explore the role of the myocardial miR-126-PI3K/AKT axis in Nar-mediated protection. The primary findings were as follows: i) Nar decreased myocardial enzyme content and enhanced cardiac tissue viability in $\mathrm{D}-\mathrm{MI} / \mathrm{R}$ rats; ii) Nar inhibited myocardial oxidative stress in D-MI/R rats; iii) Nar improved cardiac function in $\mathrm{D}-\mathrm{MI} / \mathrm{R}$ rats; and iv) Nar upregulated the myocardial miR-126-PI3K/AKT axis in D-MI/R rats. In summary, these findings revealed that Nar prevented MI/R injury by upregulating the miR-126-PI3K/AKT axis. 

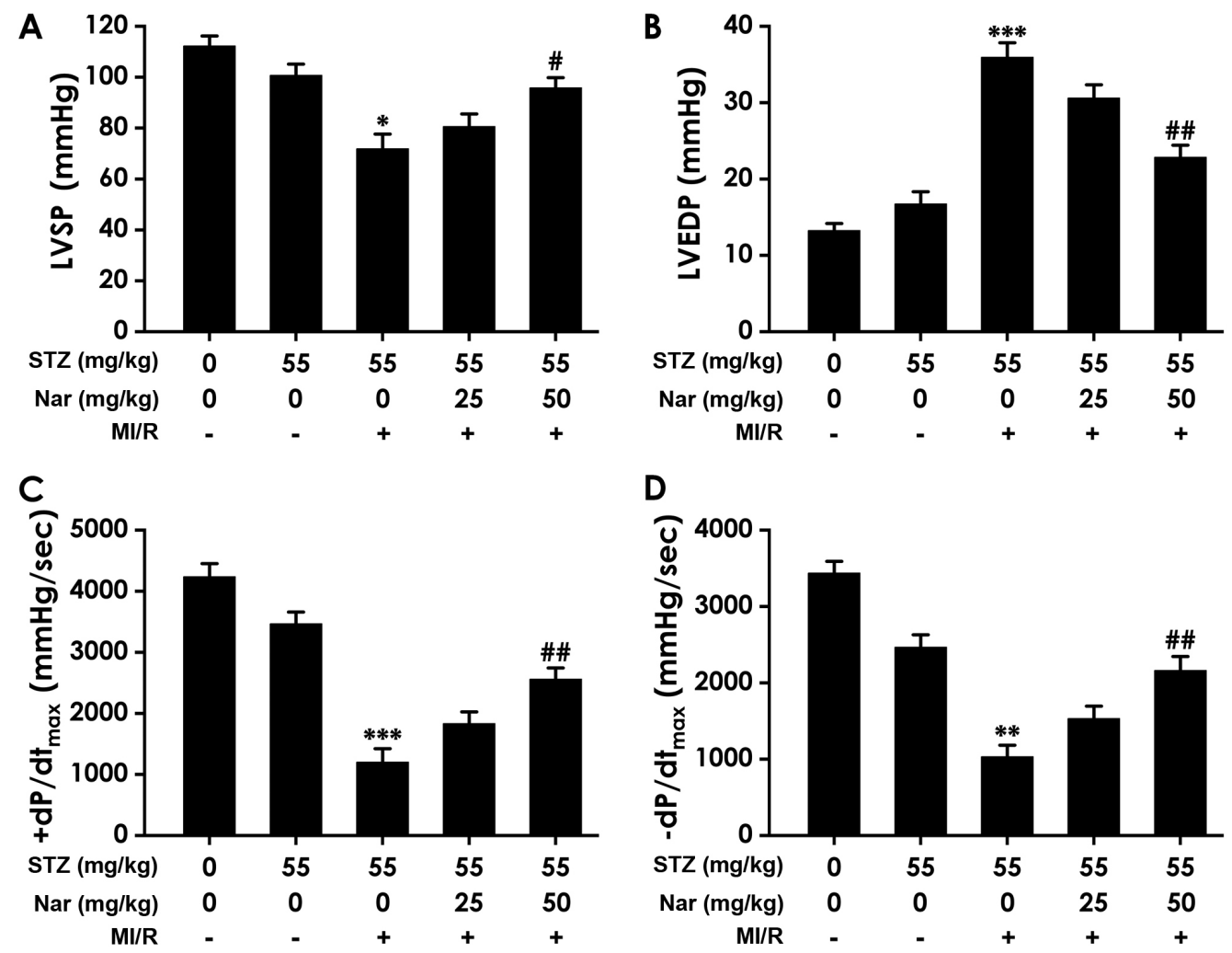

Figure 4. Effect of Nar on cardiac function in diabetic MI/R rats. (A) LVSP, (B) LVEDP, (C) $+\mathrm{dP}^{\mathrm{d}} \mathrm{dt}_{\max }$ and (D) $-\mathrm{dP} / \mathrm{dt}_{\max }$ were recorded using a hemodynamic monitoring system. Data are presented as the mean \pm SEM (number of repeated measurements: $\mathrm{n}=6-8 /$ group). ${ }^{*} \mathrm{P}<0.05,{ }^{* * *} \mathrm{P}<0.01$ and ${ }^{* * * *} \mathrm{P}<0.001$ compared with the STZ-sham group; ${ }^{\# P}<0.05$ and ${ }^{\# \#} \mathrm{P}<0.01$ compared with the STZ-M/IR group. STZ, streptozotocin; MI/R, myocardial ischemia reperfusion; Nar, naringenin; LVSP, left ventricular systolic pressure; LVEDP, left ventricular end-diastolic pressure; $+\mathrm{dP}_{\mathrm{dt}} \mathrm{m}_{\max }$, peak rate of left ventricular maximal systolic/diastolic velocity rise; - $\mathrm{dP} / \mathrm{dt}_{\max }$, peak rate of left ventricular maximal systolic/diastolic velocity fall.
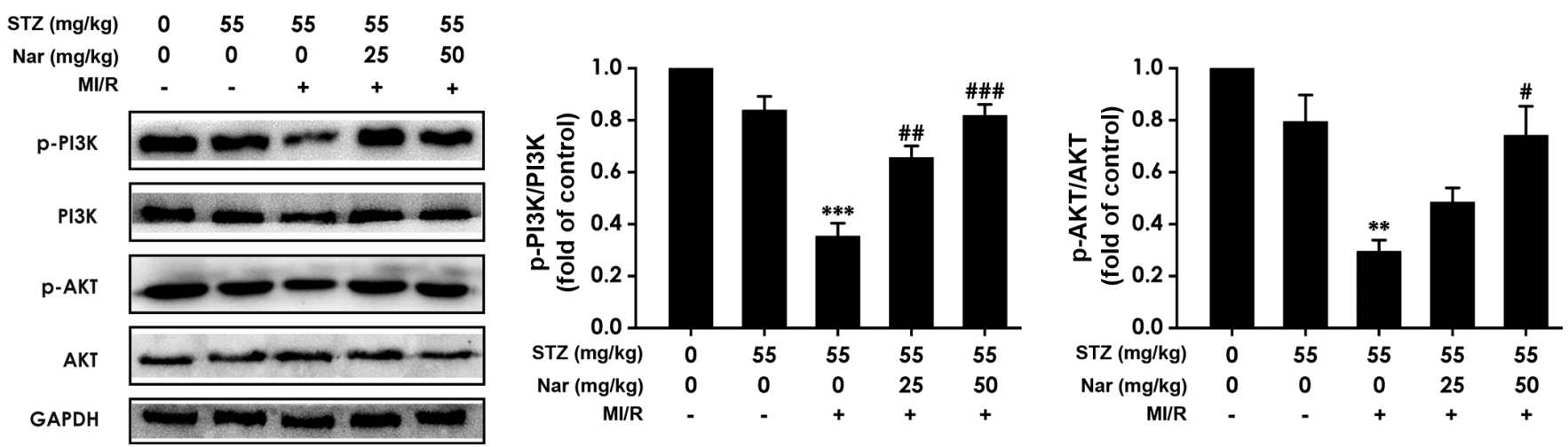

Figure 5. Effect of Nar on PI3K/AKT signaling in diabetic MI/R rats. The protein levels of p-PI3K, PI3K, p-AKT, AKT and GAPDH in myocardium were detected by western blotting. Data are presented the mean \pm SEM (number of repeated measurements: $\mathrm{n}=3 /$ group). ${ }^{* *} \mathrm{P}<0.01$ and ${ }^{* * *} \mathrm{P}<0.001$ compared with the $\mathrm{STZ}$-sham group; ${ }^{\#} \mathrm{P}<0.05$, ${ }^{\#} \mathrm{P}<0.01$ and ${ }^{\# \# \#} \mathrm{P}<0.001$ compared with the $\mathrm{STZ}-\mathrm{M} / \mathrm{IR}$ group. $\mathrm{STZ}$, streptozotocin; MI/R, myocardial ischemia reperfusion; Nar, naringenin.

In the present study, STZ-induced diabetic rats were exposed to MI/R to establish an ex vivo model of I/R injury. Myocardial enzymes, such as CK-MB and LDH, are released into the plasma during the $\mathrm{I} / \mathrm{R}$ process due to the loss of myocardial membrane integrity, and are regarded as indicators of $\mathrm{MI} / \mathrm{R}$ damage $(40,41)$. The present results show that $\mathrm{MI} / \mathrm{R}$ upregulated the levels of CK-MB and LDH of the coronary effluent following $5 \mathrm{~min}$ of reperfusion, and decreased cardiac viability in diabetic rats. Moreover, the main functional manifestation of MI/R injury is cardiac dysfunction, especially systolic and diastolic dysfunction $(42,43)$. The results of the present study showed that MI/R downregulated LVSP and $\pm \mathrm{dP} / \mathrm{dt}_{\max }$, and upregulated LVEDP in diabetic rats. Substantial evidence indicates that oxidative stress is a major contributing factor to $\mathrm{MI} / \mathrm{R}$ injury $(1,28,34,44)$. It is known that the activity of SOD and GSH-Px can be used as an objective index to evaluate the scavenging capacity of ROS (45). MDA and 8-OHdG are well-known biomarkers of lipid and DNA peroxidation, respectively, indicating lipid and DNA damage caused by excessive ROS $(46,47)$. In the present study, compared with the D-S group, D-MI/R rats had decreased activities of GSH-Px and SOD, and increased 


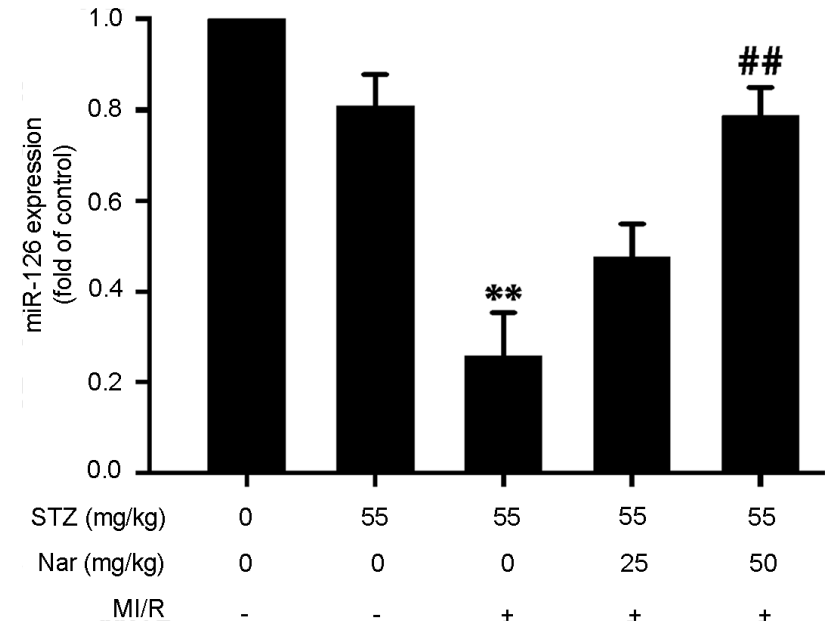

Figure 6. Effect of Nar on miR-126 expression in diabetic MI/R rats. Relative expression levels of myocardial miR-126 were determined using reverse transcription-quantitative PCR. Data are presented as the mean \pm SEM (number of repeated measurements: $\mathrm{n}=3$ /group). ${ }^{* *} \mathrm{P}<0.01$ compared with the $\mathrm{STZ}$-sham group; ${ }^{\# \prime} \mathrm{P}<0.01$ compared with the STZ-M/IR group. miR, microRNA; STZ, streptozotocin; MI/R, myocardial ischemia reperfusion; Nar, naringenin.

levels of MDA, 8-OHdG and $\mathrm{H}_{2} \mathrm{O}_{2}$. These results indicate that D-MI/R rats had upregulated levels of myocardial enzymes, reduced cardiac tissue viability, and enhanced oxidative stress and cardiac dysfunction, suggesting the successful establishment of the I/R injury model.

$\mathrm{MI} / \mathrm{R}$ injury can be triggered while treating IHD $(1,4,5)$. IHD is a leading cause of death in patients with diabetes (6). Therefore, effective approaches against D-MI/R injury may provide a better outcome in the management of diabetic IHD. It has been shown that Nar prevents MI/R injury $(19,20,39,48)$. Thus, the present study aimed to determine whether Nar protected against MI/R injury in diabetic heart. The results showed that Nar reduced the myocardial enzyme content of the coronary effluent, increased cardiac tissue viability, alleviated the oxidative stress level and improved cardiac function in D-MI/R rats. These data indicate that Nar has the ability to alleviate $\mathrm{MI} / \mathrm{R}$ injury in diabetic rats. The antioxidative effect of Nar has been confirmed in lens (49), retina (50) and renal (51) cells of diabetic rats. In addition, Nar exhibits a protective role on cardiac hypertrophy in STZ-induced diabetic mice (52). These previous findings offer a reasonable explanation for the results obtained in the present study. Therefore, it can be suggested that Nar is an effective therapeutic drug for MI/R injury in diabetic heart.

The present study also investigated the possible underlying mechanism for the protective role of Nar against D-MI/R injury. It has been shown that activation of PI3K/AKT signaling ameliorates MI/R injury (24-26). Notably, miR-126 may be a therapeutic agent for diabetes mellitus (30) and MI/R injury $(27,28)$. Therefore, it was hypothesized that the miR-126-PI3K/AKT axis may be involved in Nar-mediated protection against $\mathrm{D}-\mathrm{MI} / \mathrm{R}$ injury. The present study evaluated the role of Nar on myocardial miR-126 and $\mathrm{PI} 3 \mathrm{~K} / \mathrm{AKT}$ signaling in D-MI/R rats. It was revealed that Nar reversed the MI/R-reduced miR-126 expression level, as well as the ratios of $\mathrm{p}-\mathrm{PI} 3 \mathrm{~K} / \mathrm{PI} 3 \mathrm{~K}$ and $\mathrm{p}-\mathrm{AKT} / \mathrm{AKT}$ in $\mathrm{D}-\mathrm{MI} / \mathrm{R}$ rats, which suggested that the upregulation of the
miR-126-PI3K/AKT axis contributed to the beneficial effect of Nar on D-MI/R injury. It has previously been confirmed that the antidiabetic effect of Nar is exerted by activation of the PI3K/AKT signaling pathway (53). In addition, the activation the PI3K/AKT signaling pathway is involved in in the protection mediated by dexmedetomidine (54) and eplerenone (55) against I/R injury in the heart of diabetic rats. Furthermore, long non-coding RNA cardiac hypertrophy-related factor modulates the progression of cerebral I/R injury via the miR-126/SOX6 signaling pathway (56). These previous findings offer a reasonable explanation for the results obtained in the present study.

In conclusion, the present study demonstrated that Nar was able to prevent MI/R injury and upregulate the miR-126-PI3K/AKT axis in D-MI/R rats. These results indicated that Nar ameliorated MI/R injury by upregulating the miR-126-PI3K/AKT axis in diabetic heart, and suggest that Nar may act as a potential preventive agent for MI/R injury in diabetes, which is important for improving the therapeutic effect of diabetic IHD including antioxidant, improvement of myocardial enzyme level and enhancement of cardiac activity and function.

\section{Acknowledgements}

Not applicable.

\section{Funding}

This study was supported by the Guangdong Medical Science and Technology Research Fund Project (grant nos. A2017494 and A2016267).

\section{Availability of data and materials}

The datasets used and/or analyzed during the current study are available from the corresponding author on reasonable request.

\section{Authors' contributions}

SHL and MSW performed the experiments and data analysis. MRW was a contributor in analyzing the data and writing the manuscript. WLK contributed to the experimental design and manuscript revision. All authors read and approved the final manuscript.

\section{Ethics approval and consent to participate}

The present study was approved by the University Animal Care and Use Committee of Affiliated Hospital of Guangdong Medical (Zhanjiang, China).

\section{Patient consent for publication}

Not applicable.

\section{Competing interests}

The authors declare that they have no competing interests. 


\section{References}

1. Yellon DM and Hausenloy DJ: Myocardial reperfusion injury. N Engl J Med 357: 1121-1135, 2007.

2. Pell VR, Chouchani ET, Frezza C, Murphy MP and Krieg T: Succinate metabolism: A new therapeutic target for myocardial reperfusion injury. Cardiovasc Res 111: 134-141, 2016.

3. Whayne TF Jr: Ischemic heart disease and the Mediterranean diet. Curr Cardiol Rep 16: 491, 2014.

4. Nordlie MA, Wold LE, Simkhovich BZ, Sesti C and Kloner RA: Molecular aspects of ischemic heart disease: Ischemia/ reperfusion-induced genetic changes and potential applications of gene and RNA interference therapy. J Cardiovasc Pharmacol Ther 11: 17-30, 2006.

5. Feyzizadeh S and Badalzadeh R: Application of ischemic postconditioning's algorithms in tissues protection: Response to methodological gaps in preclinical and clinical studies. J Cell Mol Med 21: 2257-2267, 2017.

6. Orchard TJ and Costacou T: When are type 1 diabetic patients at risk for cardiovascular disease? Curr Diab Rep 10: 48-54, 2010.

7. Gharravi AM, Jafar A, Ebrahimi M, Mahmodi A, Pourhashemi E Haseli N, Talaie N and Hajiasgarli P: Current status of stem cell therapy, scaffolds for the treatment of diabetes mellitus. Diabetes Metab Syndr 12: 1133-1139, 2018

8. Whittington HJ, Babu GG, Mocanu MM, Yellon DM and Hausenloy DJ: The diabetic heart: Too sweet for its own good? Cardiol Res Pract 2012: 845698, 2012.

9. Li N, Yang YG and Chen MH: Comparing the adverse clinical outcomes in patients with non-insulin treated type 2 diabetes mellitus and patients without type 2 diabetes mellitus following percutaneous coronary intervention: A systematic review and meta-analysis. BMC Cardiovasc Disord 16: 238, 2016.

10. Lejay A, Fang F, John R, Van JA, Barr M, Thaveau F, Chakfe N, Geny B and Scholey JW: Ischemia reperfusion injury, ischemic conditioning and diabetes mellitus. J Mol Cell Cardiol 91: 11-22, 2016.

11. Jha JC, Ho F, Dan C and Jandeleit-Dahm K: A causal link between oxidative stress and inflammation in cardiovascular and renal complications of diabetes. Clin Sci (Lond) 132: 1811-1836, 2018.

12. Salehi B, Fokou PV, Sharifi-Rad M,Zucca P, Pezzani R, Martins N and Sharifi-Rad J: The therapeutic potential of naringenin: A review of clinical trials. Pharmaceuticals (Basel) 12: E11, 2019.

13. Testai L, Da Pozzo E, Piano I, Pistelli L, Gargini C, Breschi MC, Braca A, Martini C, Martelli A and Calderone V: The citrus flavanone naringenin produces cardioprotective effects in hearts from 1 year old rat, through activation of mitoBK channels. Front Pharmacol 8: 71, 2017.

14. Han X, Pan J, Ren D, Cheng $Y$, Fan $P$ and Lou $H$ Naringenin-7-O-glucoside protects against doxorubicin-induced toxicity in $\mathrm{H} 9 \mathrm{c} 2$ cardiomyocytes by induction of endogenous antioxidant enzymes. Food Chem Toxicol 46: 3140-3146, 2008.

15. Han X, Ren D, Fan P, Shen T and Lou H: Protective effects of naringenin-7-O-glucoside on doxorubicin-induced apoptosis in H9C2 cells. Eur J Pharmacol 581: 47-53, 2008.

16. Han XZ, Gao S, Cheng YN, Sun YZ, Liu W, Tang LL and Ren DM: Protective effect of naringenin-7-O-glucoside against oxidative stress induced by doxorubicin in $\mathrm{H} 9 \mathrm{c} 2$ cardiomyocytes. Biosci Trends 6: 19-25, 2012.

17. Arafa HM, Abd-Ellah MF and Hafez HF: Abatement by naringenin of doxorubicin-induced cardiac toxicity in rats J Egypt Natl Canc Inst 17: 291-300, 2005.

18. Chtourou Y, Slima AB, Makni M, Gdoura R and Fetoui H: Naringenin protects cardiac hypercholesterolemia-induced oxidative stress and subsequent necroptosis in rats. Pharmacol Rep 67: 1090-1097, 2015.

19. Yu LM, Dong X, Zhang J, Li Z, Xue XD, Wu HJ, Yang ZL, Yang $Y$ and Wang HS: Naringenin attenuates myocardial ischemia-reperfusion injury via cGMP-PKGI $\alpha$ signaling and in vivo and in vitro studies. Oxid Med Cell Longev 2019: 7670854, 2019.

20. Yu LM, Dong X, Xue XD, Zhang J, Li Z, Wu HJ, Yang ZL, Yang Y and Wang HS: Naringenin improves mitochondrial function and reduces cardiac damage following ischemia-reperfusion injury: The role of the AMPK-SIRT3 signaling pathway. Food Funct 10: 2752-2765, 2019.

21. You Q, Wu Z, Wu B, Liu C, Huang R, Yang L, Guo R, Wu K and Chen J: Naringin protects cardiomyocytes against hyperglycemia-induced injuries in vitro and in vivo. J Endocrinol 230: 197-214, 2016.
22. Yao $H$, Han $X$ and Han $X$ : The cardioprotection of the insulin-mediated PI3K/Akt/mTOR signaling pathway. Am J Cardiovasc Drugs 14: 433-442, 2014.

23. Viard P, Butcher AJ, Halet G, Davies A, Nürnberg B, Heblich F and Dolphin AC: PI3K promotes voltage-dependent calcium channel trafficking to the plasma membrane. Nat Neurosci 7: 939-946, 2004.

24. Shen D, Chen R, Zhang L, Rao Z, Ruan Y, Li L, Chu M and Zhang Y: Sulodexide attenuates endoplasmic reticulum stress induced by myocardial ischaemia/reperfusion by activating the PI3K/Akt pathway. J Cell Mol Med 23: 5063-5075, 2019.

25. Zhang BF, Jiang H, Chen J, Guo X, Li Y, Hu Q and Yang S: Nobiletin ameliorates myocardial ischemia and reperfusion injury by attenuating endoplasmic reticulum stress-associated apoptosis through regulation of the PI3K/AKT signal pathway. Int Immunopharmacol 73: 98-107, 2019.

26. Deng T, Wang Y, Wang $\mathrm{C}$ and Yan H: FABP4 silencing ameliorates hypoxia reoxygenation injury through the attenuation of endoplasmic reticulum stress-mediated apoptosis by activating PI3K/Akt pathway. Life Sci 224: 149-156, 2019.

27. Jiang C, Ji N, Luo G, Ni S, Zong J, Chen Z, Bao D, Gong X and $\mathrm{Fu}$ T: The effects and mechanism of miR-92a and miR-126 on myocardial apoptosis in mouse ischemia-reperfusion model. Cell Biochem Biophys 70: 1901-1906, 2014.

28. Wang W, Zheng Y, Wang M, Yan M, Jiang J and Li Z: Exosomes derived miR-126 attenuates oxidative stress and apoptosis from ischemia and reperfusion injury by targeting ERRFI1. Gene 690: 75-80, 2019.

29. Moghaddam AS, Afshari JT, Esmaeili SA, Saburi E, Joneidi Z and Momtazi-Borojeni AA: Cardioprotective microRNAs: Lessons from stem cell-derived exosomal microRNAs to treat cardiovascular disease. Atherosclerosis 285: 1-9, 2019.

30. Pishavar E and Behravan J: miR-126 as a Therapeutic Agent for Diabetes Mellitus. Curr Pharm Des 23: 3309-3314, 2017.

31. Naderi R, Mohaddes G, Mohammadi M, Alihemmati A, Khamaneh A, Ghyasi R and Ghaznavi R: The effect of garlic and voluntary exercise on cardiac angiogenesis in diabetes: The role of miR-126 and miR-210. Arq Bras Cardiol 112: 154-162, 2019.

32. Bhutada $P$, Mundhada $Y$, Bansod K, Bhutada C, Tawari S, Dixit $P$ and Mundhada D: Ameliorative effect of quercetin on memory dysfunction in streptozotocin-induced diabetic rats. Neurobiol Learn Mem 94: 293-302, 2010.

33. Bayrami G, Alihemmati A, Karimi P, Javadi A, Keyhanmanesh R, Mohammadi M, Zadi-Heydarabad $M$ and Badalzadeh R: Combination of vildagliptin and ischemic postconditioning in diabetic hearts as a working strategy to reduce myocardial reperfusion injury by restoring mitochondrial function and autophagic activity. Adv Pharm Bull 8: 319-329, 2018.

34. Xiao C, Xia ML, Wang J, Zhou XR, Lou YY, Tang LH, Zhang FJ, Yang JT and Qian LB: Luteolin attenuates cardiac ischemia/reperfusion injury in diabetic rats by modulating Nrf2 antioxidative function. Oxid Med Cell Longev 2019: 2719252, 2019.

35. Yu L, Liang H, Dong X, Zhao G, Jin Z, Zhai M, Yang Y, Chen W, Liu J, Yi W, et al: Reduced silent information regulator 1 signaling exacerbates myocardial ischemia-reperfusion injury in type 2 diabetic rats and the protective effect of melatonin. J Pineal Res 59: 376-390, 2015.

36. Yang JT, Qian LB, Zhang FJ, Wang J, Ai H, Tang LH and Wang HP: Cardioprotective effects of luteolin on ischemia/reperfusion injury in diabetic rats are modulated by eNOS and the mitochondrial permeability transition pathway. J Cardiovasc Pharmacol 65: 349-356, 2015.

37. Yang Y, Duan W, Jin Z, Yi W, Yan J,Zhang S, Wang N, Liang Z, Li Y, Chen W, et al: JAK2/STAT3 activation by melatonin attenuates the mitochondrial oxidative damage induced by myocardial ischemia/ reperfusion injury. J Pineal Res 55: 275-286, 2013.

38. Livak KJ and Schmittgen TD: Analysis of relative gene expression data using real-time quantitative PCR and the 2(- $\Delta \Delta \mathrm{C}(\mathrm{T}))$ method. Methods 25: 402-408, 2001.

39. Meng LM, Ma HJ, Guo H, Kong QQ and Zhang Y: The cardioprotective effect of naringenin against ischemia-reperfusion injury through activation of ATP-sensitive potassium channel in rat. Can J Physiol Pharmacol 94: 973-978, 2016.

40. Kumar A, Kaur H, Devi P and Mohan V: Role of coenzyme Q10 (CoQ10) in cardiac disease, hypertension and Meniere-like syndrome. Pharmacol Ther 124: 259-268, 2009.

41. Du L, Zhang H, Zhao H, Cheng X, Qin J, Teng T, Yang Q and Xu Z: The critical role of the zinc transporter Zip2 (SLC39A2) in ischemia/reperfusion injury in mouse hearts. J Mol Cell Cardiol 132: 136-145, 2019. 
42. Yang M, Kong DY and Chen JC: Inhibition of miR-148b ameliorates myocardial ischemia/reperfusion injury via regulation of Wnt/ß-catenin signaling pathway. J Cell Physiol 234: 17757-17766, 2019.

43. Mamamtavrishvili ND, Sharashidze NS, Abashidze RI and Kvirkveliia AA: Metabolic issues of ischemia induced myocardial dysfunction. Georgian Med News 195: 40-43, 2011 (In Russian).

44. Sinning C, Westermann D and Clemmensen P: Oxidative stress in ischemia and reperfusion: Current concepts, novel ideas and future perspectives. Biomarkers Med 11: 11031-11040, 2017.

45. Taysi S, Tascan AS, Ugur MG and Demir M: Radicals, oxidative/nitrosative stress and preeclampsia. Mini Rev Med Chem 19: 178-193, 2019.

46. Kołodziej U, Maciejczyk M, Miąsko A, Matczuk J, Knaś M, Żukowski P, Żendzian-Piotrowska M, Borys J and Zalewska A: Oxidative modification in the salivary glands of high fat-diet induced insulin resistant rats. Front Physiol 8: 20, 2017.

47. Zhao H, Zhao M, Wang Y, Li F and Zhang Z: Glycyrrhizic acid prevents sepsis-induced acute lung injury and mortality in rats. J Histochem Cytochem 64: 125-137, 2016.

48. Kara S, Gencer B, Karaca T, Tufan HA, Arikan S, Ersan I, Karaboga I and Hanci V: Protective effect of hesperetin and naringenin against apoptosis in ischemia/reperfusion-induced retinal injury in rats. ScientificWorldJournal 2014: 797824, 2014

49. Wojnar W, Zych M and Kaczmarczyk-Sedlak I: Antioxidative effect of flavonoid naringenin in the lenses of type 1 diabetic rats. Biomed Pharmacother 108: 974-984, 2018.

50. Al-Dosari DI, Ahmed MM, Al-Rejaie SS, Alhomida AS and Ola MS: Flavonoid naringenin attenuates oxidative stress, apoptosis and improves neurotrophic effects in the diabetic rat retina. Nutrients 9: E1161, 2017.
51. Roy S, Ahmed F, Banerjee S and Saha U: Naringenin ameliorates streptozotocin-induced diabetic rat renal impairment by downregulation of TGF- $\beta 1$ and IL- 1 via modulation of oxidative stress correlates with decreased apoptotic events. Pharm Biol 54: 1616-1627, 2016.

52. Zhang J, Qiu H, Huang J, Ding S, Huang B, Wu Q and Jiang Q: Naringenin exhibits the protective effect on cardiac hypertrophy via EETs-PPARs activation in streptozocin-induced diabetic mice. Biochem Biophys Res Commun 502: 55-61, 2018.

53. Nishina A, Sato D, Yamamoto J, Kobayashi-Hattori K, Hirai Y and Kimura H: Antidiabetic-like effects of naringenin-7-O-glucoside from edible chrysanthemum 'Kotobuki' and naringenin by activation of the PI3K/Akt pathway and PPAR $\gamma$. Chem Biodivers 16: e1800434, 2019.

54. Cheng X, Hu J, Wang Y, Ye H, Li X, Gao Q and Li Z: Effects of dexmedetomidine postconditioning on myocardial ischemia/reperfusion injury in diabetic rats: Role of the PI3K/Akt-dependent signaling pathway. J Diabetes Res 2018: 3071959, 2018

55. Mahajan UB, Patil PD, Chandrayan G, Patil CR, Agrawal YO, Ojha S and Goyal SN: Eplerenone pretreatment protects the myocardium against ischaemia/reperfusion injury through the phosphatidylinositol 3-kinase/Akt-dependent pathway in diabetic rats. Mol Cell Biochem 446: 91-103, 2018.

56. Gai HY, Wu C, Zhang Y and Wang D: Long non-coding RNA CHRF modulates the progression of cerebral ischemia/reperfusion injury via miR-126/SOX6 signaling pathway. Biochem Biophys Res Commun 514: 550-557, 2019. 